\title{
Differential roles of IL-1 and TNF- $\alpha$ on graft-versus-host disease and graft versus leukemia
}

\author{
Geoffrey R. Hill, ${ }^{1}$ Takanori Teshima, ${ }^{1,2}$ Armin Gerbitz, ${ }^{1}$ Luying Pan, ${ }^{1}$ Kenneth R. Cooke, ${ }^{1,2}$ \\ Yani S. Brinson, ${ }^{1}$ James M. Crawford, ${ }^{3}$ and James L.M. Ferrara ${ }^{2}$

\begin{abstract}
${ }^{1}$ Department of Pediatric Oncology, Dana Farber Cancer Institute, Harvard Medical School, Boston, Massachusetts 02115, USA ${ }^{2}$ Departments of Internal Medicine and Pediatrics, Division of Hematology and Oncology, University of Michigan Cancer Center, Ann Arbor, Michigan 48109-0560, USA

${ }^{3}$ Department of Pathology, Yale University School of Medicine, New Haven, Connecticut 06520-8023, USA
\end{abstract}

Address correspondence to: James L.M. Ferrara, Departments of Internal Medicine and Pediatrics,

Division of Hematology and Oncology, University of Michigan Cancer Center, Ann Arbor, Michigan 48109-0560, USA.

Phone: (734) 615-1340; Fax: (734) 647-9271; E-mail: ferrara@umich.edu.

Received for publication March 24, 1999, and accepted in revised form July 8, 1999.

\begin{abstract}
We demonstrate an increase in graft-versus-host disease (GVHD) after experimental bone marrow transplant (BMT) when cyclophosphamide (Cy) is added to an otherwise well-tolerated dose (900 cGy) of total body irradiation (TBI). Donor $\mathrm{T}$ cell expansion on day +13 was increased after conditioning with $\mathrm{Cy} / \mathrm{TBI}$ compared with Cy or TBI alone, although cytotoxic T lymphocyte (CTL) function was not altered. Histological analysis of the gastrointestinal tract demonstrated synergistic damage by Cy/TBI and allogeneic donor cells, which permitted increased translocation of LPS into the systemic circulation. TNF- $\alpha$ and IL-1 production in response to LPS was increased in BMT recipients after Cy/TBI conditioning. Neutralization of IL-1 significantly reduced serum LPS levels and GVHD mortality, but it did not affect donor CTL activity. By contrast, neutralization of TNF- $\alpha$ did not prevent GVHD mortality but did impair CTL activity after BMT. When P815 leukemia cells were added to the bone marrow inoculum, allogeneic BMT recipients given the TNF- $\alpha$ inhibitor relapsed at a significantly faster rate than those given the IL-1 inhibitor. To confirm that the role of TNF- $\alpha$ in graft versus leukemia (GVL) was due to effects on donor $\mathrm{T}$ cells, cohorts of animals were transplanted with $\mathrm{T}$ cells from either wild-type mice or p55 TNF- $\alpha$ receptor-deficient mice. Recipients of TNF- $\alpha$ p 55 receptor-deficient $T$ cells demonstrated a significant impairment in donor CTL activity after BMT and an increased rate of leukemic relapse compared with recipients of wild-type $T$ cells. These data highlight the importance of conditioning in GVHD pathophysiology, and demonstrate that TNF- $\alpha$ is critical to GVL mediated by donor T cells, whereas IL-1 is not.
\end{abstract}

J. Clin. Invest. 104:459-467 (1999).

\section{Introduction}

Allogeneic bone marrow transplantation (BMT) remains the treatment of choice for a number of malignant conditions. Much of the therapeutic potential of this procedure for these diseases relates to the graft-versusleukemia (GVL) effect, which eradicates host malignancy after BMT and is mediated by donor $\mathrm{T}$ and natural killer (NK) cells (1). GVL effects are closely associated, however, with graft-versus-host disease (GVHD), which remains the major limitation of allogeneic BMT. During acute GVHD, cytokine dysregulation occurs as a consequence of synergistic interactions between cells of both myeloid and lymphoid lineages (2). T cells present in the donor inoculum encounter allogeneic histocompatibility antigens on host tissues and, in the presence of IL-12, secrete the Th1 cytokines IFN- $\gamma$ and IL-2 (3). IFN- $\gamma$ primes monocytes and macrophages to secrete large quantities of inflammatory cytokines after stimulation by LPS (4). Together with NK cells and cytoxic T lymphocytes (CTLs), these cytokines mediate GVHD target organ damage (5). Depletion of T cells from the graft effectively prevents GVHD, but it results in the loss of the GVL effect and increases the rate of graft failure (6).
An alternative approach to the prevention of acute GVHD is to retain mature T cells in the bone marrow graft but to disrupt the amplification of inflammatory cytokine effectors. With this in mind, randomized clinical trials using novel cytokine inhibitors that neutralize TNF- $\alpha$ and IL- 1 are currently in progress as adjuncts to GVHD prophylaxis.

Until recently, GVHD has been thought to occur independently of BMT conditioning. However, clinical studies have shown a correlation between GVHD incidence and total body irradiation (TBI) dose $(7,8)$ and conditioning regimens containing TBI compared with those containing only chemotherapy (9). The incidence of conditioning-related toxicity has also been associated with GVHD severity (10). We have recently confirmed that large doses of TBI increase GVHD severity by amplifying the dysregulation of inflammatory cytokines (11). TBI and allogeneic immune cells synergize to damage the gastrointestinal (GI) tract, thereby permitting increased translocation of LPS into the systemic circulation. This damage, together with increased production of TNF- $\alpha$ by host cells after TBI, leads to an increase in the morbidity and mortality of GVHD. 
Because standard BMT conditioning regimens contain both cyclophosphamide (Cy) and TBI, we were interested in the contribution of Cy to subsequent GVHD. We therefore chose to examine this issue in a murine BMT model, where the MHC disparity between donor and host could be tightly controlled and the clinical variable of post-transplant immunosuppression could be avoided. Our results confirm that $\mathrm{Cy} / \mathrm{TBI}$ conditioning amplifies both the cellular and inflammatory effectors of GVHD. Notably, IL-1, rather than TNF- $\alpha$, is a principal mediator of GVHD mortality after conditioning with $\mathrm{Cy} / \mathrm{TBI}$. Furthermore, neutralization of IL-1 improves leukemia-free survival by preserving GVL effects while inhibiting GVHD. Surprisingly, neutralization of TNF- $\alpha$ did not prevent GVHD after conditioning with Cy/TBI but did inhibit the GVL effect. Further experiments confirmed that donor $\mathrm{T}$ cells require TNF- $\alpha$ to generate optimal CTL activity after BMT. Thus, neutralization of TNF- $\alpha$ can impair the GVL effect.

\section{Methods}

Mice. Female C57BL/6 (B6, H-2 $\left.{ }^{\mathrm{b}}, \mathrm{Ly}-5.2^{+}\right)$and B6D2F1 $\left(\mathrm{H}-2^{\mathrm{b} / \mathrm{d}}, \mathrm{Ly}-5.2^{+}\right)(12)$ mice were purchased from The Jackson Laboratory (Bar Harbor, Maine, USA). B6 Ly-5a $\left(\mathrm{H}-2^{\mathrm{b}}, \mathrm{Ly}-5.1^{+}\right)(12)$ mice were purchased from Frederick Cancer Research Facility (Frederick, Maryland, USA). B6 mice deficient in the TNF p55 receptor were supplied by Immunex (Seattle, Washington, USA) and have been described previously (13-16). The age of mice used as BMT recipients ranged between 9 and 15 weeks. Mice were housed in sterilized microisolator cages and received filtered water and normal chow, or autoclaved hyperchlorinated drinking water for the first 3 weeks after BMT.

$B M T$. Mice were transplanted according to a standard protocol described previously $(11,17)$. On days -2 and -1 , recipient mice received $100 \mathrm{mg} / \mathrm{kg}$ of Cy by intraperitoneal injection. On day 0 , more than 30 hours after the last Cy dose, mice received 900 cGy TBI $\left({ }^{137} \mathrm{Cs}\right.$ source, dose rate $107 \mathrm{cGy} / \mathrm{min}$ ), split into 2 doses separated by 3 hours to minimize GI toxicity. A total of $5 \times 10^{6}$ bone
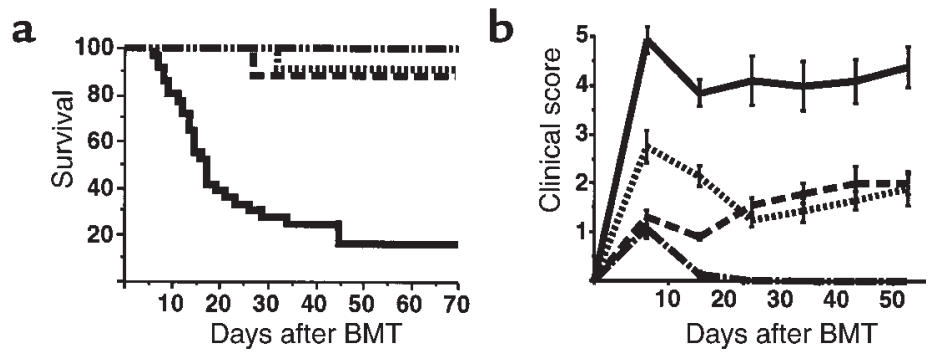

\section{Figure 1}

GVHD severity is significantly increased after a $\mathrm{Cy} / \mathrm{TBI}$ conditioning regimen. B6D2F1 recipients received allogeneic $\mathrm{B} 6$ donor bone marrow and T cells after 1 of 3 conditioning regimens: Cy (dotted line; $n=11$ ), TBI (900 cGy; dashed line; $n=10$ ), or $\mathrm{Cy} / \mathrm{TBI}$ (solid line; $n=35$ ). Syngeneic recipients received B6D2F1 bone marrow and $\mathrm{T}$ cells after $\mathrm{Cy} / \mathrm{TBI}$ (dashed line broken by dots; $n=15$ ). (a) Mortality was significantly $(P<0.0001)$ greater in allogeneic Cy/TBI group than in all others. (b) GVHD severity was determined by clinical score (as described in Methods) and was significantly greater $(P<0.001)$ after $\mathrm{Cy} / \mathrm{TBI}$ conditioning than after all other regimens. Allogeneic groups were significantly different $(P<0.001)$ from syngeneic recipients from day 14 forward. Results are from 3 similar experiments. marrow cells and $2 \times 10^{6}$ nylon wool-purified splenic donor T cells were resuspended in $0.25 \mathrm{~mL}$ of Leibovitz's L-15 media (GIBCO BRL, Gaithersburg, Maryland, USA) and injected intravenously into recipients. In GVL eximents, $5 \times 10^{4}$ P815 leukemia cells $\left(\mathrm{H}-2^{\mathrm{d}}\right)$ were experiments, B6Ly-5 $5^{\mathrm{a}}\left(\mathrm{H}-2^{\mathrm{b}}\right.$, Ly-5.1 $\left.{ }^{+}\right)$animals were used as donors. Survival was monitored daily; recipients' ody weights and GVHD clinical score were measured examining percentage of Ly-5.1 $1^{+}$cells in peripheral lood at various times after transplantation.

h TNFR:Fc and anti-IL-1R treatment. Recombinant human immunoglobulin (rhTNFR:Fc) and hamster anti-mouse type I IL-1 receptor (anti-IL-1R-M147) were pplied and used in doses recommended by Immunex. were injected intraperitoneally with TNFR:Fc (100 $2,-1$, and 0 and then on alternate days up to and cluding day 16 (12 injections total). Mice in control mmunoglobulin.

Assessment of GVHD. The degree of systemic GVHD was ssessed by a scoring system that sums changes in 5 clinparameters: weight loss, posture (hunching), activi(18). Individual mice were ear tagged and graded weekly rom 0 to 2 for each criterion without knowledge of ment group. conjugated) CD8 were purchased from PharMingen (San Diego, California, USA). F4/80 antibody specific for incubated with mAb $2.4 \mathrm{G} 2$ for 15 minutes at $4{ }^{\circ} \mathrm{C}$ and with the relevant FITC- or PE-conjugated $\mathrm{mAb}$ for 30 minutes at $4^{\circ} \mathrm{C}$. Finally, cells were washed twice with PBS/0.2\% BSA, fixed with PBS/1\% paraformaldehyde, and analyzed by FACScan (Becton Dickinson Immunocytometry Systems, San Jose, California, USA). In engraftment studies, the percentage of donor cells was determined by the percentage of peripheral blood cells positive for Ly-5.1 or lacking H2-Dd.

Cell cultures. All culture media and incubation conditions were as described previously $(11,17)$. Peritoneal macrophages were lavaged on day 7 after BMT and pooled from 3-4 individual animals within a group before culture at $10^{5}$ cells per well in flat-bottomed 96-well Falcon plates (Becton Dickinson Co., Lincoln Park, New Jersey, USA) with or without LPS. Supernatants were collected at 4 hours for TNF- $\alpha$ and at 48 hours for day 7 IL-1 $\beta$ analysis by ELISA. Splenocytes were removed from animals 14 days after transplant, and 3-6 spleens were combined from each group. Single-cell suspensions were then layered over Ficoll- 
$\mathbf{a}$
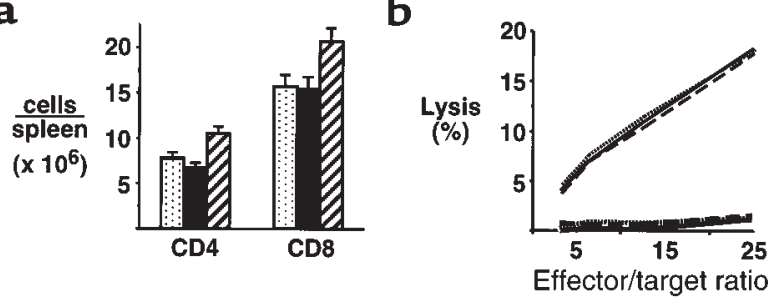

Figure 2

$\mathrm{Cy} / \mathrm{TBI}$ conditioning accelerates T-cell expansion. (a) Thirteen days after $\mathrm{BMT}$, the numbers of $\mathrm{CD} 4^{+}$and $\mathrm{CD} 8^{+}$cells were determined in the spleens of allogeneic BMT recipients after conditioning with $\mathrm{Cy}$ (dotted bars; $n=9$ ), TBI (filled bars; $n=10$ ), or $\mathrm{Cy} / \mathrm{TBI}$ (hatched bars; $n=13$ ). $\mathrm{CD}^{+}$and $\mathrm{CD} 8^{+}$numbers $\left(\times 10^{6}\right)$ in syngeneic BMT recipients after $\mathrm{Cy} / \mathrm{TBI}(n=6)$ were $3.2 \pm 0.7$ and $1.8 \pm 0.3$, respectively $(P<0.03$ for $\mathrm{Cy} / \mathrm{TBI}$ vs. $\mathrm{Cy}$ and $\mathrm{TBI}$ allogeneic and $\mathrm{Cy} / \mathrm{TBI}$ syngeneic groups). (b) $\mathrm{CTL}$ activity of splenic $T$ cells 13 days after BMT subsequent to conditioning with Cy (dotted line), TBI (dashed line), or Cy/TBI (solid line). Spleens from 3-4 animals in each allogeneic group were pooled and used as effectors in standard 5-hour ${ }^{51} \mathrm{Cr}$ release assays against host-type P815 targets (top curves) or donor-type EL4 targets (bottom curves). Results represent average curves from 3 similar experiments.

Paque (Amersham Pharmacia Biotech, Piscataway, New Jersey, USA) and centrifuged at $800 \mathrm{~g}$ for 15 minutes. Cells were collected from the interface and washed twice before suspension in supplemented 10\% FCS/RPMI. Cells were plated in 96-well flat-bottomed plates (Falcon) at a concentration of $2 \times 10^{5} \mathrm{~T}$ cells $\left(\mathrm{CD}^{+}\right.$plus $\left.\mathrm{CD}^{+}\right)$per well with $10^{5}$ irradiated (20 Gy) peritoneal macrophages lavaged from naive B6D2F1 (allogeneic) or B6 (syngeneic) animals. At 40 hours, cultures were pulsed with $\left[{ }^{3} \mathrm{H}\right]$ thymidine $(1 \mu \mathrm{Ci} /$ well $)$, and proliferation was determined 20 hours later on a 1205 Betaplate reader (Wallac Oy, Turku, Finland).

Cytokine ELISAs. The antibodies used in the TNF- $\alpha$ and IL- $1 \beta$ assays were purchased from Genzyme Pharmaceuticals (Cambridge, Massachusetts, USA). Antibodies used in the IFN- $\gamma$, IL-2, and IL-4 assays were purchased from PharMingen. All assays were performed according to the manufacturer's protocol. For TNF- $\alpha$ and IL-1 $\beta$ production by macrophages, results were expressed as units per $10^{5} \mathrm{~F} 4 / 80^{+}$cells (units per fraction of $\mathrm{F} 4 / 80^{+}$peritoneal cells).

${ }^{51} \mathrm{Cr}$ release assays. A total of $2 \times 10^{6}$ P815 (H-2d $)$ or EL4 (H-2 $)$ tumor targets were labeled with $100 \mu \mathrm{Ci}$ of ${ }^{51} \mathrm{Cr}$ for 2 hours. After washing 3 times, labeled targets were plated at $10^{4}$ cells per well in U-bottom plates (Corning-Costar Corp., Cambridge, Massachusetts, USA). Splenocytes from allogeneic BMT recipients (prepared as already described here) were added to quadruplicate wells at varying effector/target ratios and incubated for 5 hours. Maximal and background release were deter- mined by the addition of Triton-X (Sigma Chemical Co., St. Louis, Missouri, USA) or media alone, respectively. ${ }^{51} \mathrm{Cr}$ activity in supernatants taken 5 hours later were determined in a Cobra auto gamma counter (Packard Instrument Co., Meriden, Connecticut, USA), and lysis was expressed as a percentage of maximum. Spleen lytic units were defined as the number of $\mathrm{T}$ cells per spleen $\left(\mathrm{CD} 4^{+}\right.$ and $\mathrm{CD}^{+}$) divided by the number of effectors needed to lyse $20 \%$ of targets, as described previously (19).

Serum LPS estimation. For determination of endotoxin concentration in serum, the Limulus amebocyte lysate (LAL) assay (BioWhittaker Inc., Walkersville, Maryland, USA) was performed according to the manufacturer's protocol. Briefly, serum samples were collected and analyzed using pyrogen-free materials, diluted 10-fold ( $\mathrm{vol} / \mathrm{vol}$ ) in LAL reagent water, and heated to $70^{\circ} \mathrm{C}$ for 5 minutes to remove any nonspecific inhibition to the assay. Samples were then incubated with equal volumes of LAL for 10 minutes at $37^{\circ} \mathrm{C}$ and developed with equal volumes of substrate solution for 6 minutes. The absorbance of the assay plate was read at $405 \mathrm{~nm}$ using the same microplate reader used in cytokine assays. Samples and standards were run in duplicate, and the lower limit of detection was $0.15 \mathrm{U} / \mathrm{mL}$. All units expressed are relative to the US reference standard EC-6.

Histology. Formalin-preserved distal small bowel was embedded in paraffin, and $5-\mu \mathrm{m}$-thick sections were stained with hematoxylin and eosin for histological examination. Slides were coded and examined in a blinded fashion by 1 individual (J.M. Crawford), using a semiquantitative scoring system for abnormalities known to be associated with GVHD $(11,20)$. Specifically, 7 parameters each were scored (villus blunting, crypt regeneration, crypt epithelial cell apoptosis, crypt loss, luminal sloughing of cellular debris, lamina propria inflammatory cell infiltrate, and mucosal ulceration) using a system that denoted 0 as normal; 0.5 as focal and rare; 1 as focal and mild; 2 as diffuse and mild; 3 as diffuse and moderate; and 4 as diffuse

\section{a}
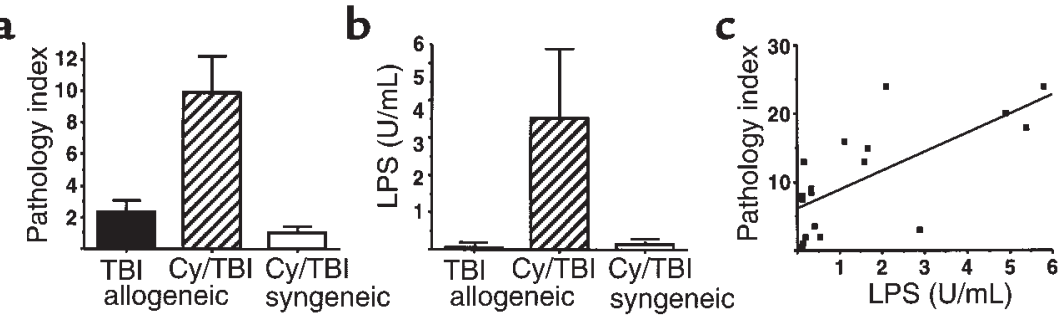

Figure 3

$\mathrm{Cy} / \mathrm{TBI}$ conditioning enhances GVHD of the Gl tract and subsequent endotoxemia. (a) Gl tract injury was determined 7 days after BMT by a semiquantitative histological scoring system as described in Methods. The severity of injury was significantly increased $(P<0.02)$ in allogeneic BMT recipients after $\mathrm{Cy} / \mathrm{TBI}$ conditioning (hatched bars; $n=13$ ) compared with recipients transplanted with syngeneic marrow after $\mathrm{Cy} / \mathrm{TBI}$ conditioning (open bars; $n=6$ ) or allogeneic recipients transplanted after TBI alone (filled bars; $n=8$ ). (b) Serum LPS levels 7 days after BMT were significantly increased $(P<0.03)$ in allogeneic BMT recipients after $\mathrm{Cy} / \mathrm{TBI}$ conditioning $(n=17)$ compared with recipients transplanted with syngeneic marrow after Cy/TBI conditioning $(n=9)$ or allogeneic recipients transplanted after TBI alone $(n=23)$. (c) Elevated levels of serum LPS 7 days after BMT correlate with the severity of GI tract injury $(P<0.01)$. Cy/TBI allogeneic: filled squares $(n=13)$; Cy/TBI syngeneic: filled circles $(n=3)$; TBI allogeneic: open circles $(n=2)$. 
Table 1

Engraftment (percentage of donor cells) in allogeneic BMT recipients

\begin{tabular}{lcccc}
\hline Day & Cy & TBI & Cy/TBI & Inhibitor \\
$28-40$ & $92.8 \pm 4.0$ & 100 & 100 & None \\
& ND & ND & 100 & TNFR:FC \\
& ND & ND & 100 & Anti-IL-1R \\
70 & $96.7 \pm 1.4$ & 100 & ND & None
\end{tabular}

Engraftment of donor cells in the peripheral blood was determined by FACS analysis of Ly- 5 alleles or the absence of $\mathrm{H}-2 \mathrm{~d}$ cells (for $\mathrm{Cy} / \mathrm{TBI}$ recipients), as detailed in Methods. ND, not done.

and severe, as published previously in studies of human $(21,22)$ and experimental $(11,20)$ GVHD histology. Scores were added to provide a total (maximum score was thus 28).

Statistical analysis. Survival curves were plotted using Kaplan-Meier estimates. The Mann-Whitney $U$ test was used for the statistical analysis of cytokine data, LPS levels clinical scores, weight loss, and histology, whereas the Mantel-Cox log rank test was used to analyze survival data. Statistical significance was set at $P<0.05$.

\section{Results}

The addition of Cy to TBI increases GVHD mortality and morbidity. We have previously reported that the intensity of TBI used in BMT conditioning regimens has an important role in determining the severity of subsequent acute GVHD (11). Because TBI is commonly used clinically in conjunction with $\mathrm{Cy}$, we studied the effect of a conditioning regimen using both of these agents on GVHD to both MHC and minor histocompatibility antigens in a well-described murine model (B6 $\rightarrow$ B6D2F1). Cy was given in clinically relevant doses $(100 \mathrm{mg} / \mathrm{kg}$ on days -2 and -1 ), and the TBI dose (900 cGy on day 0 ) was chosen because when used alone it was known to permit complete hematopoietic engraftment and induce only mild GVHD (11). Animals were transplanted after 1 of 3 conditioning regimens: $\mathrm{Cy}$ alone, $\mathrm{TBI}$ alone, or $\mathrm{Cy} / \mathrm{TBI}$. As shown in Figure 1a, less than $20 \%$ of allogeneic BMT recipients conditioned with $\mathrm{Cy} / \mathrm{TBI}$ survived at day 70 . Clinical signs of GVHD in surviving animals were quantified by summing the scores ( $0-2)$ for each of 5 parameters: mobility, posture, fur texture, skin integrity, and weight loss, as reported previously (18). GVHD was severe (clinical scores $>4$ ) in allogeneic animals transplanted after conditioning with Cy/TBI (Figure 1b). Survival in allogeneic BMT recipients conditioned with either $\mathrm{Cy}$ or TBI alone was $90 \%$ at day 70 . GVHD in these animals was mild (clinical scores $<2$ ), confirming that the increase in GVHD after conditioning with Cy/TBI was a consequence of the conditioning regimen itself. As expected, all syngeneic BMT recipients $(\mathrm{B} 6 \mathrm{D} 2 \mathrm{~F} 1 \rightarrow \mathrm{B} 6 \mathrm{D} 2 \mathrm{~F} 1)$ transplanted after $\mathrm{Cy} / \mathrm{TBI}$ survived, and clinical scores in these animals returned to baseline by day 14 .

The increased severity of GVHD after Cy/TBI confirms observations made in a fully allogeneic BMT model $(\mathrm{B} 6 \rightarrow \mathrm{B} 10 . \mathrm{BR})$ in which death from GVHD occurred more rapidly after Cy/TBI conditioning than after TBI alone (23). We also investigated this phenomenon in a third strain combination of GVHD to minor $\mathrm{H}$ antigens.
In the $\mathrm{B} 10 . \mathrm{BR} \rightarrow \mathrm{CBA}$ model, long-term survival was significantly decreased after Cy/TBI compared with TBI alone (2/10 vs. $12 / 18 ; P<0.01)$, and clinical GVHD scores on day 28 in the Cy/TBI group were significantly greater $(5.8 \pm 0.42$ vs. $3.4 \pm 0.41 ; P=0.006)$. In an $\mathrm{LP} \rightarrow \mathrm{B} 6$ model using a lower dose of radiation (800 cGy), clinical GVHD was again significantly greater in animals conditioned with Cy/TBI $(n=32)$ than with TBI alone $(n=20)$ (day 35 scores: $3.6 \pm 0.6$ vs. $1.2 \pm 0.2 ; P<0.0001)$. Thus, the increased GVHD caused by the addition of Cy to the conditioning was not limited to a particular donor or recipient mouse strain, nor to particular histocompatibility differences between donor and recipient.

We next evaluated donor engraftment because mixed donor/host chimerism has been associated with tolerance of donor $\mathrm{T}$ cells to host tissues and reduced GVHD (24). Donor engraftment was documented between days 28 and 40 and at day 70 in allogeneic recipients by phenotyping of $\mathrm{Ly}-5$ or $\mathrm{H}-2^{\mathrm{d}}$ alleles, as detailed in Methods. All allogeneic BMT recipients $(n=$ 4-6) studied after Cy/TBI or TBI conditioning had $100 \%$ donor cells in the peripheral blood by day 28 (Table 1), excluding mixed chimerism as a cause for the reduction in GVHD after conditioning with TBI alone. Animals transplanted after Cy had persistent residual host cells in the peripheral blood at days 28 and 70 (Table 1), consistent with the inability to achieve complete myeloablation with Cy alone.

Cy/TBI conditioning accelerates donor T-cell expansion. Donor $\mathrm{T}$ cells are critical in the induction of acute GVHD (5); therefore, we studied the effect of Cy/TBI conditioning on T-cell expansion and function after allogeneic BMT. Splenic T-cell expansion was maximal at day 13 after BMT, and the number of $\mathrm{CD}^{+}$and $\mathrm{CD}^{+}$ cells in the spleen was significantly increased in BMT recipients transplanted after $\mathrm{Cy} / \mathrm{TBI}$ conditioning compared with either Cy or TBI conditioning alone (Figure 2a). $\mathrm{CD}^{+}$and $\mathrm{CD}^{+}$cell numbers in allogeneic groups were increased 2- to 3 -fold and 8- to 12 -fold, respectively, over levels seen in syngeneic BMT recipients (data not shown). Donor CTL function of splenic T cells was also assessed by analysis in standard ${ }^{51} \mathrm{Cr}$ lysis assays. At day 13, donor T cells from allogeneic groups were equivalent in their ability to lyse host-type cells (Figure 2b). Taken together, these data suggest that the addition of Cy to TBI-containing conditioning regimens accelerates the expansion of donor $\mathrm{T}$ cells responding to host alloantigens. Although the lytic function of donor T cells was not increased on a per-cell basis, the increased absolute number of donor $\mathrm{T}$ cells after Cy/TBI conditioning was likely to increase the damage inflicted on host tissues by T-cell effectors, consistent with the increased severity of acute GVHD after Cy/TBI conditioning.

Cy/TBI conditioning amplifies early GI tract injury, systemic LPS levels, and inflammatory cytokine responses to LPS. We have recently demonstrated the critical role of the GI tract in amplifying the inflammatory component of acute $\operatorname{GVHD}(11,17)$. We were therefore interested in the effect of Cy/TBI conditioning for allogeneic BMT on GI tract injury. Using a previously described semiquantitative scoring index $(11,17)$ in which features of GI damage were scored on a scale of $0-4$ and summed (see Methods), we 
compared the degree of injury in the small bowel of allogeneic BMT recipients after $\mathrm{Cy} / \mathrm{TBI}$ and TBI conditioning. The small bowel of syngeneic BMT recipients transplanted after Cy/TBI conditioning served as non-GVHD control. As shown in Figure 3a, the addition of Cy to an otherwise well-tolerated TBI dose dramatically enhanced damage to the small bowel. This effect was due to the synergistic interaction of both $\mathrm{Cy} / \mathrm{TBI}$ conditioning and allogeneic donor effector cells, as injury in syngeneic BMT recipients transplanted after $\mathrm{Cy} / \mathrm{TBI}$ conditioning was minimal. LPS is a constituent of the bacterial wall of normal bowel flora, and, as such, the presence and level of LPS in the systemic circulation may serve as a marker for GI tract injury $(11,25)$. In confirmation of the GI tract pathology, LPS levels were significantly elevated in allogeneic BMT recipients transplanted after $\mathrm{Cy} / \mathrm{TBI}$ conditioning (Figure $3 \mathrm{~b}$ ). Serum levels of LPS correlate with the degree of GI tract injury $(P<0.01$; Figure $3 \mathrm{c})$.

LPS is a potent stimulus for inflammatory cytokine production, and we next studied the response of macrophages from BMT recipients to LPS in vitro 7 days after BMT. Interestingly, both TNF- $\alpha$ and IL- $1 \beta$ production to LPS were significantly higher in allogeneic $\mathrm{BMT}$ recipients transplanted after $\mathrm{Cy} / \mathrm{TBI}$ conditioning than after either Cy or TBI alone (Figure 4). These data suggested that in addition to the effects on donor T cells, $\mathrm{Cy} / \mathrm{TBI}$ conditioning might also amplify the inflammatory component of acute GVHD.

$I L-1$, rather than TNF- $\alpha$, is an important mediator of GVHD mortality after Cy/TBI conditioning. Having demonstrated an increase in both TNF- $\alpha$ and IL-1 production in vitro after $\mathrm{Cy} / \mathrm{TBI}$ conditioning, we next studied the contribution of each of these cytokines to GVHD mortality in vivo. These studies used rhTNFR:Fc, a bivalent soluble form of the TNF- $\alpha$ receptor bound to the heavy portion of human immunoglobulin molecule, which was administered to animals intraperitoneally on days $-3,-2,-1$, and 0 and then on alternate days up to and including day 16. We have previously shown this agent to be effective in preventing GVHD mortality when used in this schedule after TBI conditioning alone $(11,25)$. In a second group of BMT recipients, we neutralized IL-1 using the hamster anti-mouse IL-1 receptor antibody (anti-IL-1R) in the same dosing schedule. Control groups received the same doses of human or hamster IgG. Surprisingly, TNF- $\alpha$ blockade did not prevent GVHD mortality (Figure 5a), whereas IL-1 inhibition did (Figure 5b). Neither of these agents impaired engraftment, which remained $100 \%$ donor (Table 1). To study the potential mechanisms of the relative benefit of IL- 1 inhibition, LPS levels were measured 7 days after BMT. As seen in Table 2, inhibition of IL-1 (but not TNF- $\alpha$ ) significantly protected the GI tract, as measured by the reduced serum LPS levels. In contrast, splenic T-cell expansion 14 days after BMT was not affected by either inhibitor, and proliferation and cytokine production by donor cells to host-type stimulators in mixed lymphocyte culture (MLC) were similarly unaffected. Interestingly, there was a trend $(P=$ $0.06)$ toward a reduction in the spleen lytic activity only in recipients of the TNF- $\alpha$ inhibitor (Table 2). This reduction in the capacity to lyse host cells was not associated with a reduction in the severity of GVHD.
Table 2

LPS levels and T-cell responses to alloantigen

\begin{tabular}{lccc}
\hline & Control immunoglobulin & TNFR:Fc & Anti-IL-1R \\
LPS & $1.7 \pm 0.4$ & $1.1 \pm 0.2$ & $0.5 \pm 0.2^{\mathrm{A}}$ \\
T-cell expansion & $11.2 \pm 1.2$ & $14.2 \pm 2.2$ & $10.5 \pm 2.0$ \\
MLC & & & \\
Stimulation index & $5.1 \pm 0.2$ & $4.8 \pm 0.6$ & $3.7 \pm 0.5$ \\
IFN-g & $2.1 \pm 0.3$ & $2.5 \pm 0.2$ & $2.9 \pm 0.2$ \\
IL-2 & $0.7 \pm 0.1$ & $0.7 \pm 0.1$ & $0.7 \pm 0.1$ \\
IL-4 & $1.0 \pm 0.4$ & $0.8 \pm 0.3$ & $0.4 \pm 0.1$ \\
CTLs & & & \\
Spleen LU $_{20}$ & $91 \pm 7$ & $56 \pm 17^{\mathrm{B}}$ & $98 \pm 8$ \\
\hline
\end{tabular}

LPS (mean \pm SEM) was determined in the sera of allogeneic BMT recipients 7 days after BMT $(n=8-10)$. T-cell expansion (million cells per spleen) was determined in the spleen of allogeneic BMT recipients by FACS analysis $(n=13-16)$. Data were combined from 2 experiments and represent mean \pm SEM. Spleens were then pooled from 4-6 animals per group and used as effectors in ${ }^{51} \mathrm{Cr}$ assays and MLC, as outlined in Methods. Stimulation index is the proliferation to host antigens/proliferation to donor antigens. CTL results are expressed as $L_{20}$ per spleen (the number of spleen T cells per the number of these T cells required to lyse 20\% of host-type P815 targets). Results represent mean \pm SEM from 3 similar experiments. Cytokines and LPS are in $\mathrm{U} / \mathrm{mL}$. ${ }^{\mathrm{A} P}<0.05$ vs. control and TNFR:Fc. ${ }^{\mathrm{B} P}=0.06$ vs. control.

IL-1 inbibition improves leukemia-free survival after Cy/TBI conditioning, whereas TNF- $\alpha$ inhibition is associated with increased leukemic relapse. In light of the apparent impairment in CTL generation in BMT recipients receiving the TNF- $\alpha$ inhibitor, it was of interest to study the impact of such agents on the GVL effect after allogeneic BMT. Animals were transplanted, as described above, with the addition of $5 \times 10^{4}$ host-type P815 leukemic cells $\left(\mathrm{H}-2^{\mathrm{d}}\right)$ to the donor inoculum. Animals received TNF- $\alpha$ or IL- 1 inhibition, as described above, with either TNFR:Fc or anti-IL-1R, respectively. The presence of hepatic and/or splenic nodules at postmortem, or hindlimb paralysis with spinal infiltration of tumor at postmortem, was deemed a leukemia-related death. Syngeneic BMT recipients treated with control human IgG and transplanted with P815 all died from leukemia (median day of relapse: 13; range: 11-22). As seen previously, IL-1 inhibition significantly delayed GVHD mortality in allogeneic ani-
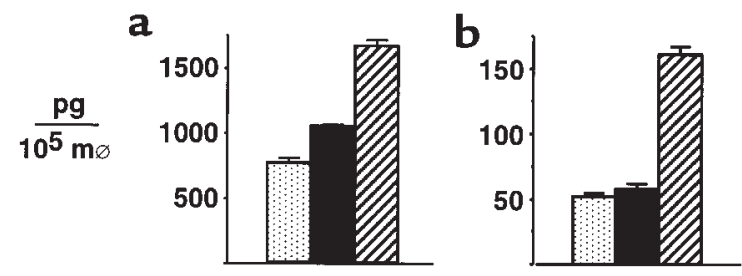

\section{Figure 4}

$\mathrm{Cy} / \mathrm{TBI}$ conditioning amplifies macrophage TNF- $\alpha$ and IL- $1 \beta$ responses to LPS. Peritoneal macrophages were pooled from 4 animals per group and stimulated in vitro with $0.1 \mu \mathrm{g} / \mathrm{mL}$ of LPS. TNF- $\alpha(\mathbf{a})$ and IL- $1 \beta$ (b) were determined in the supernatant by ELISA at 4 and 48 hours, respectively, after conditioning with Cy (stippled bars), TBI (filled bars), or Cy/TBI (hatched bars). Results are expressed as mean \pm SD of quadruplicate wells and represent 1 of 3 similar experiments. Macrophages from syngeneic BMT recipients transplanted after $\mathrm{Cy} / \mathrm{TB}$ I conditioning produced $484 \pm$ 41 pg of TNF- $\alpha$ and $8 \pm 1$ pg of IL- $1 \beta . P<0.05$ for Cy/TBI vs. Cy and TBI. 
a

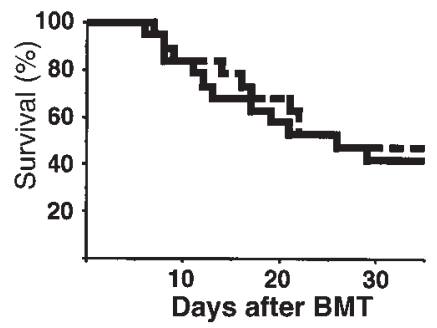

b

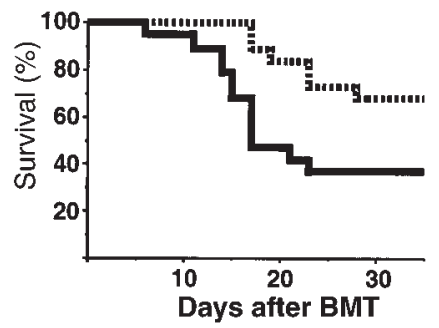

$\mathrm{TNFR}^{-/} \mathrm{T}$ cells died from leukemia, whereas less than $50 \%$ of BMT recipients of wildtype allogeneic $\mathrm{T}$ cells did so. These data confirm that TNF- $\alpha$ signaling through the T-cell p55 TNFR is required for optimal CTL generation and subsequent GVL activity after allogeneic BMT, and that blockade of TNF- $\alpha$ impaired a GVL effect.

\section{Discussion}

This study demonstrates an increase in GVHD severity after allogeneic BMT subsequent to the addition of $\mathrm{Cy}$ to an otherwise well-tolerated TBI dose (900 cGy). This increase in GVHD was associated with an increase in donor cytotoxic T-cell expansion and the induction of IL1 -mediated mortality that was induced both by greater sensitivity of macrophages to LPS and higher circulating LPS levels caused by GI tract damage in allogeneic $\mathrm{BMT}$ recipients conditioned with $\mathrm{Cy} / \mathrm{TBI}$. This increase in serum LPS levels was pre-

mals, which translated to improved overall survival by day 70 (Figure 6a). The rate of leukemic relapse was significantly higher in recipients of the TNF- $\alpha$ inhibitor than in those treated with the IL- 1 inhibitor $(P<0.05)$.

The T-cell $p 55$ TNF- $\alpha$ receptor is required for optimal CTL generation and GVL activity after allogeneic BMT. The lysis of allogeneic cells in CTL assays may be achieved through a number of pathways, including perforin and FasL, and by cytokines such as TNF- $\alpha$ and IFN- $\gamma(26)$. Because the P815 leukemia cell line is MHC class I positive and class II negative, the principal pathway of rejection is likely to be by $\mathrm{CD}^{+}$cells, which preferentially use the perforin pathway (26), a finding we have recently confirmed in vivo (27) Given our surprising data, we were therefore interested in whether TNF- $\alpha$ is required after BMT in order to optimally generate anti-host CTL effector function. To study this question, we used T cells from knockout B6 mice that lacked the p55 TNF- $\alpha$ receptor ( $p 55$ $T_{N F R^{-/}}$) (13-16). Animals were transplanted with wildtype bone marrow and either wild-type T cells or $p 55$ $\mathrm{TNFR}^{-1-} \mathrm{T}$ cells. Fourteen days later, the spleens from animals transplanted with $p 55 \mathrm{TNFR}^{-/-} \mathrm{T}$ cells had a $60 \%$ reduction in lytic activity compared with those from recipients of wild-type T cells (Figure 7a). T cells from animals in which the $p 75 \mathrm{TNFR}^{-/-}$was similarly deleted by homozygous recombination (13) generated normal spleen lytic function after BMT (data not shown), confirming a requirement for TNF- $\alpha$ signaling in CTL generation through the p55 TNFR. To study the relevance of these findings in vivo, GVL activity was determined in animals transplanted with wild-type bone marrow and wild-type or $p 55$ TNFR $^{-/-}$T cells in combination with P815 leukemia, as described in Methods. As shown in Figure 7b, 95\% of BMT recipients of allogeneic p55
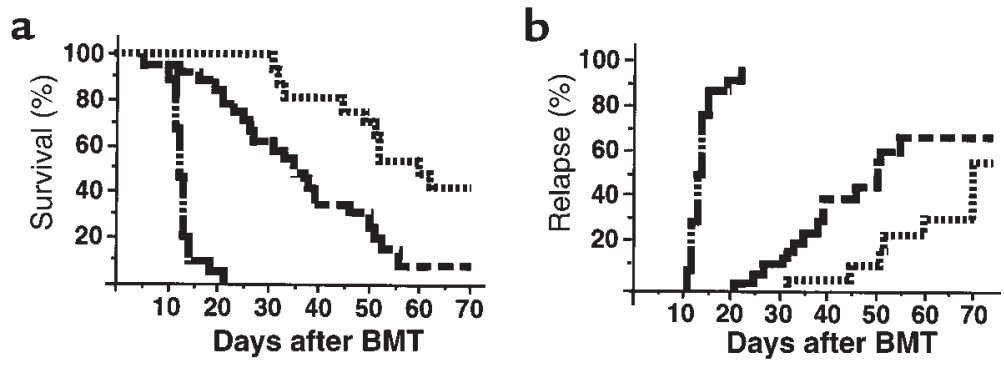

\section{Figure 6}

TNF- $\alpha$ blockade impairs GVL activity after allogeneic BMT. Allogeneic BMT recipients were transplanted with TNF (dashed line; $n=30$ ) or IL-1 blockade (dotted line; $n=19$ ) as in Figure 5, with the addition of $5 \times 10^{4}$ host-type P815 leukemic cells to the donor inoculum. Syngeneic BMT recipients (dashed line broken by dots; $n=15$ ) were used as positive controls. (a) Overall survival; (b) leukemic relapse. $P<0.002$ and $P<0.05$ for survival and relapse between allogeneic arms. 
Figure 7

Optimal CTL generation after allogeneic BMT requires TNF- $\alpha$ signaling through the TNF p55 receptor. (a) Spleen lytic activity $\left(\mathrm{LU}_{20}\right)$ was determined 14 days after BMT in animals transplanted with wild-type T-cell-depleted bone marrow and either wild-type T cells (hatched bar) or p55 TNFR-1- T cells (solid bar). Results are from 3 experiments. $P<0.05$ between groups. (b) The rate of relapse was determined after allogeneic BMT with $5 \times 10^{4}$ P815 cells and wild-type T-cell-depleted bone marrow and wild-type $T$ cells (solid line; $n=30$ ), or wild-type T-cell-depleted bone marrow and $p 55$ TNFR- - - T cells (dashed line; $n=20)$. $P<0.001$ between groups. a

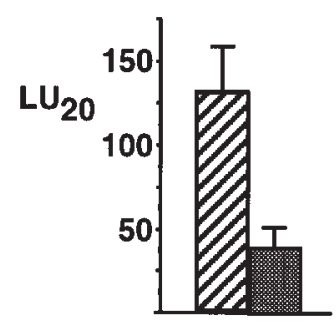

b

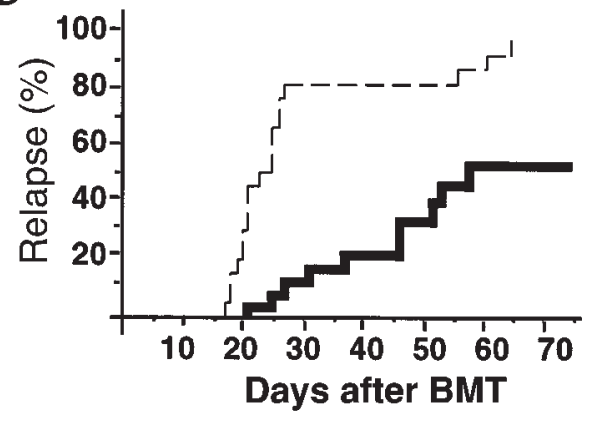

a synergistic reduction in host anti-donor activity in these BMT recipients. In clinical BMT, the numbers of residual host mononuclear cells after Cy/TBI is some 10-fold lower than after conditioning regimens containing only chemotherapy (e.g., busulfan and Cy) (31). Our results suggest that this reduction may play a role in the increased severity of GVHD seen after the former regimen (9).

The ability of Cy to mediate organ toxicity $(32,33)$ and induce production of IL-1 (34) and IFN- $\gamma(35)$ has been described previously. In addition, cytotoxic agents such as $\mathrm{Cy}$ increase endothelial reactivity and the expression of adhesion molecules through the induction of IL-1 (36). Inflammatory cytokines are also known to be important in the pathogenesis of experimental acute GVHD (11,37-40) and clinical acute GVHD, in which both TNF- $\alpha$ (41) and IL-1 (42) appear to play critical roles. It is of particular interest to compare the results of this study with the dramatic benefit of TNF- $\alpha$ blockade on survival and GI tract injury in allogeneic BMT recipients after intensive conditioning regimens containing large TBI doses $(11,25)$. Despite an increase in host macrophage TNF- $\alpha$ and IL- $1 \beta$ production to LPS in allogeneic BMT recipients after Cy/TBI, blockade of IL-1, rather than TNF- $\alpha$, reduced GVHD mortality and serum LPS levels. The protection afforded by IL-1 neutralization suggests that this cytokine is a dominant mediator of GI tract damage induced by the combination of $\mathrm{Cy} / \mathrm{TBI}$ and allogeneic donor cells. The dominant role of cytokines, rather than cellular effectors, in GVHD of the GI tract is consistent with previous findings from this laboratory $(11,25)$ and others $(37,43)$. However, these studies have focused on conditioning regimens using TBI alone in which GI tract injury is clearly mediated by TNF- $\alpha$. To our knowledge, this study is the first to show a causal relationship between GI tract injury mediated by IL- 1 and conditioning with Cy/TBI.

Although inflammatory cytokines generated by the conditioning regimen clearly amplify GVHD, they do not in and of themselves cause mortality (syngeneic Cy/TBI controls show 100\% survival), and the histocompatibility differences between donor and recipient remain the dominant predictive factors of acute GVHD. The inflammation caused by the "cytokine storm" of acute GVHD (44) amplifies the damage to host tissues mediated by donor CTLs. Both of the major lytic pathways used by CTLs - perforin and FasL - enhance acute GVHD in experimental models, and the Fas pathway appears to be the predominant mediator of hepatic GVHD $(45,46)$.

A surprising conclusion of these studies was the requirement of TNF- $\alpha$ for an optimal GVL effect. This requirement has recently been observed independently in a similar experimental GVL model (47). TNF- $\alpha$ is secreted by cytotoxic $T$ cells and macrophages and can act directly as an effector pathway in target cell killing (26). TNF- $\alpha$ is also required for the optimal generation of human CTLs in vitro and, when added to the sensitizing phase of the primary MLC, is associated with a selective upregulation of IL-2R on $\mathrm{CD}^{+} \mathrm{T}$ cells (48). Conversely, IL-1 enhances $\mathrm{T}$ cell-dependent immune responses by amplifying the function of dendritic cells (49), and IL-1 receptor antagonists do not inhibit CTL responses in vivo (50). TNF- $\alpha$ can also increase granzyme activity induced by IL-2, as determined by benzyloxycarbonyl-L-lysine thiobenzylester-esterase (BLT-E) activity (47). In the mouse, TNF- $\alpha$ has been described as an important inhibitor of TGF- $\beta$ that inhibits the generation of CTLs but not their effector function (51). It is possible that the increased production of TGF- $\beta$ observed in BMT recipients after Cy conditioning (23) makes the generation of CTLs after Cy/TBI conditioning more dependent on TNF- $\alpha$ than after conditioning with regimens containing TBI alone. Despite the putative role of TNF- $\alpha$ in CTL generation and function in vitro, its role in vivo has not been established, particularly in the setting of GVHD. Our data clearly demonstrate that the neutralization of TNF- $\alpha$ after BMT impairs CTL activity, although the generation of $\mathrm{CD}^{+}$ cells is not impaired (Table 2). This impairment would be consistent with a reduction in granzyme activity predicted by Robinet et al. (48). This defect was also seen after transplantation of donor $\mathrm{T}$ cells lacking the p55 TNF- $\alpha$ receptor (Figure 7), although the use of these cells also impaired the generation of donor CTLs (data not shown). This additional deficit may relate to the more complete blockade of TNF- $\alpha$ afforded by the use of p55 TNFR ${ }^{-1-} \mathrm{T}$ cells. Together, these data confirm the critical role of TNF- $\alpha$ in the optimal generation and function of CTLs after allogeneic BMT. Donor CTLs are critical mediators of GVL after allogeneic BMT (52), and our results confirm that neutralization of TNF- $\alpha$ compromises GVL effects.

Our findings suggest that Cy and TBI preferentially induce an IL-1-dominant inflammatory cytokine pro- 
file during GVHD and, consistent with our previous work, suggest that this process may be interrupted at a number of steps within the cytokine cascade. The efficacy of systemic TNF- $\alpha$ and IL-1 neutralization after clinical allogeneic BMT may reduce GVHD severity, but the dominant role of each cytokine is likely to be heavily determined by the conditioning regimen, and blockade of both cytokines may be required. Furthermore, our data suggest that TNF- $\alpha$ blockade is likely to impair GVL. Alternatively, there may be benefit in replacing Cy with other, less toxic immunosuppressive agents such as fludarabine, although the effect of such maneuvers on the induction of GVHD will need to be studied. For these reasons, the addition of IL- 1 inhibition to agents that strengthen the GI mucosal barrier before conditioning, such as IL-11 (17) or keratinocyte growth factor (53), may be preferable. These cytokine shields act more proximally in the cytokine cascade, and prevent the translocation of LPS and other immunostimulatory molecules from the GI tract into the systemic circulation. The combination of IL-1 neutralization and these cytokines has the potential to allow the more intensive conditioning schedules desired to control malignancy without an increase in GVHD. Because this approach does not completely neutralize TNF- $\alpha$ or alter anti-host CTL generation, the desirable GVL effects after allogeneic BMT are likely to be maintained.

1. Truitt, R., Johnson, B., and McCabe, C. 1997. Graft versus leukemia. Marcel Dekker Inc.. New York, NY. 385-424.

2. Krenger, W., Hill, G., and Ferrara, J. 1997. Cytokine cascades in acute graft-versus-host disease. Transplantation. 64:553-558.

3. Kichian, K., Nestel, F.P., Kim, D., Ponka, P., and Lapp, W.S. 1996. IL12 p40 messenger RNA expression in target organs during acute graft-versus-host disease. J. Immunol. 157:2851-2856.

4. Nestel, F.P., Price, K.S., Seemayer, T.A., and Lapp, W.S. 1992. Macrophage priming and lipopolysaccharide-triggered release of tumor necrosis factor alpha during graft-versus-host disease. J. Exp. Med. 175:405-413.

5. Hill, G.R., Krenger, W., and Ferrara, J.L.M. 1997. The role of cytokines in acute graft-versus-host disease. Cytokines Cell. Mol. Ther. 3:257-265.

6. Ferrara, J.L.M., and Deeg, H.J. 1991. Graft versus host disease. N. Engl. J. Med. 324:667-674.

7. Nash, A., et al. 1992. Acute graft-versus-host disease analysis of risk factors after allogeneic marrow transplantation and prophylaxis with cyclosporine and methotrexate. Blood. 80:1838-1845.

8. Clift, R.A., et al. 1990. Allogeneic marrow transplantation in patients with acute myeloid leukemia in first remission: a randomized trial of two irradiation regimens. Blood. 76:1867-1871.

9. Clift, R.A., et al. 1994. Marrow transplantation for chronic myeloid leukemia: a randomized study comparing cyclophosphamide and total body irradiation with busulfan and cyclophosphamide. Blood. 84:2036-2043

10. Deeg, H.J., Spitzer, T.R., Cottler-Fox, M., Cahill, R., and Pickle, L.W. 1991. Conditioning-related toxicity and acute graft-versus-host disease in patients given methotrexate/cyclosporine prophylaxis. Bone Marrow Transplant. 7:193-198.

11. Hill, G.R., et al. 1997. Total body irradiation effects on acute graft versus host disease. The role of gastrointestinal damage and inflammatory cytokines. Blood. 90:3204-3213.

12. Morse, H.C., Shen, F.W., and Hammerling, U. 1987. Genetic nomenclature for loci controlling mouse lymphocyte antigens. Immunogenetics. 25:71-78.

13. Page, D.M., Roberts, E.M., Peschon, J.J., and Hedrick, S.M. 1998. TNF receptor-deficient mice reveal striking differences between several models of thymocyte negative selection. J. Immunol. 160:120-133.

14. Pfeffer, K., et al. 1993. Mice deficient for the $55 \mathrm{kD}$ tumor necrosis factor receptor are resistant to endotoxin shock, yet succumb to $L$. monocytogenes infection. Cell. 73:457-467.

15. Erickson, S., et al. 1994. Decreased sensitivity to tumour-necrosis factor but normal T-cell development in TNF receptor-2-deficient mice. Nature. 372:560-563.

16. Peschon, J.J., et al. 1998. TNF receptor-deficient mice reveal divergent roles for p55 and p75 in several models of inflammation. J. Immunol. 160:943-952.

17. Hill, G.R., et al. 1998. Interleukin-11 promotes T cell polarization and prevents acute graft-versus-host disease after allogeneic bone marrow transplantation. J. Clin. Invest. 102:115-123.

18. Cooke, K.R., et al. 1996. An experimental model of idiopathic pneumonia syndrome after bone marrow transplantation. I. The roles of minor $\mathrm{H}$ antigens and endotoxin. Blood. 8:3230-3239.

19. Su, H., Leite-Morris, K., Braun, L., and Biron, C. 1991. A role for transforming growth factor-beta 1 in regulating natural killer and $\mathrm{T}$ lymphocyte proliferative responses during acute infection with lymphocytic choriomeningitis virus. J. Immunol. 147:2717-2727.

20. Blatter, D.D., Crawford, J.M., and Ferrara, J.L.M. 1990. Nuclear magnetic resonance of hepatic graft-versus-host disease in mice. Transplantation. 50:1011-1018.

21. Snover, D.C., et al. 1985. A histopathological study of gastric and small intestinal graft-versus-host disease following allogeneic bone marrow transplantation. Hum. Pathol. 16:387-392.

22. Snover, D.C., Weisdorf, S.A., Ramsay, N.K., McGlave, P., and Kersey, J.H. 1984. Hepatic graft versus host disease: a study of the predictive value of liver biopsy in diagnosis. Hepatology. 4:123-130.

23. Panoskaltsis-Mortari, A., et al. 1997. The critical early proinflammatory events associated with idiopathic pneumonia syndrome in irradiated murine allogenic recipients are due to donor $\mathrm{T}$ cell infusion and potentiated by cyclophosphamide. J. Clin. Invest. 100:1015-1027.

24. Sykes, M. 1996. Chimerism and central tolerance. Curr. Opin. Immunol. 8:694-703.

25. Cooke, K., et al. 1998. Tumor necrosis factor- $\alpha$ production to lipopolysaccaride stimulation by donor cells predicts the severity of experimental acute graft-versus-host disease. J. Clin. Invest. 102:1882-1891

26. Shresta, S., Pham, C., Thomas, D., Braubert, T., and Ley, T. 1998. How do cytotoxic lymphocytes kill their targets? Curr. Opin. Immunol. 10:581-587.

27. Teshima, T., et al. 1999. IL-11 separates graft-versus-leukemia effects from graft-versus-host disease after bone marrow transplantation. $J$. Clin. Invest. 104:317-325.

28. Ballas, Z. 1986. Lymphokine-activated filler (LAK) cells. I. Differential recovery of LAK, natural killer cells, and cytotoxic T lymphocytes after a sublethal dose of cyclophosphamide. J. Immunol. 137:2380-2384.

29. Lehnert, S., and Rybka, W.B. 1994. Amplification of the graft-versushost reaction by cyclophosphamide: dependence on timing of drug administration. Bone Marrow Transplant. 13:473-477.

30. Merluzzi, V. 1985. Comparison of murine lymphokine-activated killer cells, natural killer cells, and cytotoxic T lymphocytes. Cell Immunol. 95:95-104.

31. Papadakis, V., Mackinnon, V., and Kernen, N. 1994. Differential effect of pre-transplant cytoreduction on recovery of day zero host circulating cells. Bone Marrow Transplant. 14:623-630.

32. Cailleau, C., Diu-Hercend, A., Ruuth, E., Westwood, R., and Carnaud, C. 1997. Treatment with neutralizing antibodies specific for IL-1 beta prevents cyclophosphamide-induced diabetes in nonobese diabetic mice. Diabetes. 46:937-940.

33. Kachel, D., and Martin, W. 1994. Cyclophosphamide-induced lung toxicity: mechanism of endothelial injury. J. Pharmacol. Exp. Ther. 268:42-46.

34. Exon, J., Henningsen, G., Koller, L., and Talcott, P. 1986. The selectivity of isoprinosine, NPT 15392, avridine and cyclophosphamide on multiple immune responses in rats. Int. J. Immunopharmacol. 8:53-62.

35. Schwaighofer, H., et al. 1996. Serum levels of cytokines and secondary messages after T-cell-depleted and non-T-cell-depleted BMT: influence of conditioning and hematopoietic reconstitution. Transplantation. 62:947-953.

36. Bertomeu, M., et al. 1990. Chemotherapy enhances endothelial cell reactivity to platelets. Clin. Exp. Metastasis. 8:511-518.

37. Piguet, P.F., Grau, G.E., Allet, B., and Vassalli, P.J. 1987. Tumor necrosis factor/cachectin is an effector of skin and gut lesions of the acute phase of graft-versus-host disease. J. Exp. Med. 166:1280-1289.

38. Wall, D.A., and Sheehan, K.C. 1994. The role of tumor necrosis factor-alpha and interferon gamma in graft-versus-host disease and related immunodeficiency. Transplantation. 57:273-279.

39. Shalaby, M.R., Fendly, B., Sheehan, K.C., Schreiber, R.D., and Ammann, A.J. 1989. Prevention of the graft-vs-host reaction in newborn mice by antibodies to tumor necrosis factor-alpha. Transplantation. 47:1057-1061.

40. Abhyankar, S., Gilliland, D.G., and Ferrara, J.L.M. 1993. Interleukin 1 is a critical effector molecule during cytokine dysregulation in graft-versus-host disease to minor histocompatibility antigens. Transplant. 56:1518-1523.

41. Holler, E., et al. 1995. Modulation of acute graft-verses-host disease 
after allogeneic bone marrow transplantation by tumor necrosis factor alpha (TNF-alpha) release in the course of pretransplant conditioning: role of conditioning regimens and prophylactic application of a monoclonal antibody neutralizing human TNF-alpha (MAK 195F). Blood. 86:890-899.

42. Antin, J.H., et al. 1994. Recombinant human interleukin-1 receptor antagonist in the treatment of steroid-resistant graft-versus-host disease. Blood. 84:1342-1348.

43. Hattori, K., et al. 1998. Differential effects of anti-fas ligand and antitumor necrosis factor a antibodies on acute graft-versus-host disease pathologies. Blood. 91:4051-4055.

44. Antin, J.H., and Ferrara, J.L.M. 1992. Cytokine dysregulation and acute graft-versus-host disease. Blood. 80:2964-2968.

45. Braun, Y.M., et al. 1996. Cytotoxic T cells deficient in both functional Fas ligand and perforin show residual cytolytic activity yet lose their capacity to induce lethal acute graft-versus-host disease. J. Exp. Med. 183:657-661.

46. Baker, M.B., Altman, N.H., Podack, E.R., and Levy, R.B. 1996. The role of cell-mediated cytotoxicity in acute GVHD after MHC-matched allogeneic bone marrow transplantation in mice. J. Exp. Med. 183:2645-2656.

47. Tsukada, N., Kobata, T., Aizawa, Y., Yagita, H., and Okumura, K. 1999. Graft-versus-leukemia effect and graft-versus-host disease can be differentiated by cytotoxic mechanisms in a murine model of allogeneic bone marrow transplantation. Blood. 93:2738-2747.

48. Robinet, E., et al. 1990. Evidence for tumor necrosis factor- $\alpha$ involvement in the optimal induction of class I allospecific cytotoxic T cells. J. Immunol. 144:4555-4561.

49. Koide, S., Inaba, K., and Steinman, R. 1987. Interleukin 1 enhances T-dependent immune responses by amplifying the function of dendritic cells. J. Exp. Med. 165:515-530.

50. Faherty, D.A., et al. 1992. Failure of IL-1 receptor antagonist and monoclonal anti-IL-1 receptor antibody to inhibit antigen-specific immune responses in vivo. J. Immunol. 148:766-771.

51. Ranges, G., Figari, I., Espevik, T., and Palladino, M. 1987. Inhibition of cytotoxic T cell development by transforming growth factor beta and reversal by recombinant tumor necrosis factor. J. Exp. Med. 166:991-998.

52. Nash, R.A., and Storb, R. 1996. Graft-versus-host effect after allogeneic hematopoietic stem cell transplantation: GVHD and GVL. Curr. Opin. Immunol. 8:674-680.

53. Panoskaltsis-Mortari, A., Lacey, D., Vallera, D., and Blazer, B. 1998. Keratinocyte growth factor administered before conditioning ameliorates graft-versus-host disease after allogeneic bone marrow transplantation in mice. Blood. 92:3960-3967. 